\title{
Kızılötesi Isıtma ile Polistiren Latekslerin Polimer Kompozit Filmlerindeki Film Oluşumuna Etkisi
}

\author{
Önder Yarg1 ${ }^{1 *}$ \\ ${ }^{1 *}$ Yıldız Teknik Üniversitesi, Fen Edebiyat Fakültesi, Fizik Bölümü, İstanbul, Türkiye, (ORCID: 0000-0001-6343-0057), oyargi@yildiz.edu.tr
}

(Ilk Geliş Tarihi Aralık 2020 ve Kabul Tarihi Ocak 2021)

(DOI: 10.31590 /ejosat.838833)

ATIF/REFERENCE: Yarg1, Ö. (2021). Kızılötesi Isıtma ile Polistiren Latekslerin Polimer Kompozit Filmlerindeki Film Oluşumuna. Avrupa Bilim ve Teknoloji Dergisi, (22), 13-18.

$\ddot{O} \mathbf{z}$

Bu çalışma, polistiren (PS)-poliakrilamid (PAAm) ve polistiren (PS)-Na Alginat (Alg) kompozitlerinden film oluşumunun UV-Visible tekniğgi kullanılarak incelendiği spektroskopik bir teknik sunmaktadır. Tüm numuneler, (PS)/poliakrilamid (PAAm) ve polistiren (PS) /Na-Alginat (Alg) filmlerinin farklı bileşimleriyle bir cam altlık üzerine damlatma yöntemiyle oda sıcaklığında hazırlandı. Kızılötesi ısıtma ile tavlanan filmlerin boşluk kapanması ve interdifüzyon aşamaları için karşılık gelen aktivasyon enerjileri hesaplandı. Kızılötesi 1sıtma ile tavlanan Polistiren (PS)/Na-Alginat (Alg) kompozit filmler için boşluk kapatma işlemleri için hesaplanan enerjilerin polistiren (PS)/poli akrilamid (PAAm) kompozit filmlerden daha küçük olduğu bulundu.

Anahtar Kelimeler: Polistren, Poliakrilamid, Film Oluşumu.

\section{Effect of Polystrene Latexes on the film formation of Polymer Composite Films by Infrared Heating}

\begin{abstract}
This work presents a spectroscopic technique that film formation from polystyrene (PS)-polyacrylamide (PAAm) and polystyrene (PS)/Na-Alginate (Alg) composites was investigated using by UV-Visible technique. All samples were prepared at room temperature by casting methode on a glass substrate with different compositions of (PS)/polyacrylamide (PAAm) and polystyrene (PS)/NaAlginate (Alg) films. Corresponding activation energies for the void closure and the interdiffusion stages were calculated of the films annealed by infrared heating. It was found that the calculated energies for the void closure processes smaller for the polystyrene (PS)/Na-Alginate (Alg) composite films than that of polystyrene (PS)/polyacrylamide (PAAm) composite films by infrared heating.
\end{abstract}

Keywords: Polystrene, Polyacrylamide, Film Formation.

\footnotetext{
*Sorumlu Yazar: oyargi@yildiz.edu.tr
} 


\section{Giriş}

Polimerler, toz kaplama uygulamalarında yakın kızılötesi aralığında 1şı̆̆ı etkili bir şekilde emer [1]. Bir çalışma, fırın ve Kızılötesi ısıtma arasında, kurutma süresini belirlemek için sulu poli (vinil alkol) çözeltilerine bu iki ısıtma türünü uygulamıştır. Sadece firın da kurutma için bu süre 120 dakika sürmüştür. Bununla birlikte, konvektif ve IR radyasyon birleştirildiğinde, aynı PVA [2] ile kuruma süresi 15 dakikaya düşmüştür. Lateksler çoğunlukla suda koloidal bir dispersiyon oluşturur. Kuruma sıcaklığı sirasıyla yumuşak ve sert lateksler olarak bilinen cam geçiş sıcaklığı, $\mathrm{T}_{\mathrm{g}}$ nin altında veya üzerindeyse lateksler yumuşak ya da sert lateksler olarak anılırlar. Lateks film oluşumu ise şu şekilde açıklanır: Islak aşama olan ilk aşamadır, bu aşamada lateksler çözelti içinde sulu formdadırlar. Çözücünün buharlaşması ile parçacıkların birbirine yakın paketlenmiş bir şekil oluşturduğu ikinci aşamaya gelinir. Kullanılan lateks, sert lateks ise oluşan deformasyonu minimum film oluşumu için gerekli olan sıcaklığın üzerinde boşlukların kapanmasına [3,4] neden olur ve daha sonra boşluklar kapandıktan sonra son olarak homojen bir film oluşumu için [4] fazla ısıtma ile parçacık-parçacık sınırları boyunca interdifüzyon süreci başlar $[5,6]$. Bu aşamada sınırlar kalkmış olur ve karşılıklı zincir geçişleri başlar. Latekslerden film oluşumunu açıklamak için literatür de birçok farklı çalışma türleri mevcuttur: elektron [7] atomik kuvvet mikroskop [8], nötron saçılması[9], 1şımasız enerji transferi [10] ve kararlı durum floresans (SSF) [11]. Elipsometri [12], foton geçirgenliği (PT) [13-15] yansıma [16] taramalı elektron mikroskop (SEM) [17], [18-20] elektron
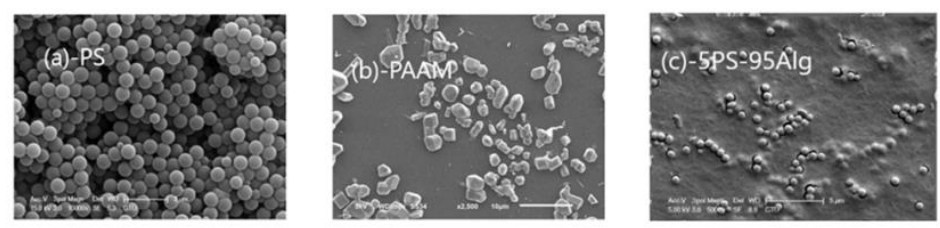

Şekil 1: (a) saf PS (b) saf PAAM ve (c) 5 PS -\% 95 Alg kompozit filmlerin SEM görüntüsü.

\section{Araştırma Sonuçları ve Tartışma}

PS/Alg ve PS/PAAM kompozit filmler için 1şık geçirgenliği $\mathrm{I}_{\text {tr }}$ nin 800 nm'de sıcaklığa bağımlı değişimi sırasıyla Şekil 2 ve Şekil 3 'te sunulmuştur. mikroskopları ve lateks sistemlerinin karakterizasyonu ile ilgili çalışmalar sunulmuştur.

$\mathrm{Bu}$ çalışmada PS/Alg ve PS/PAAM kompozitlerinin film oluşumu kızılötesi 1sıtma kullanılarak UV-Visible tekniği ile incelenmiştir. İki deney setinin aktivasyon enerjileri boşluk kapatma ve difüzyon arası modellerden hesaplandı. Boşluk kapanma enerjilerinin PS/Alg için azaldığı, PS/PAAM filmleri için ise arttığ1 görüldü. Örneklerden gelen geçirgenlik, her iki kompozit setleri içinde $800 \mathrm{~nm}$ 'de olarak bulundu.

\section{Materyal ve Metot}

\subsection{PS, PAAM ve Alginat}

Stiren monomerin polimerizasyon işlemi Alfa Asea'den satın alınmıştır. (Potasyum persülfat) PS lateksleri için KPS başlatıcı hazırlandı. KPS deiyonize suda çözüldü ve ardından $90^{\circ} \mathrm{C}$ 'de çözüye eklenmiştir. Çözeltide, PolyFluorTM 511 Polysciences'dan temin edilen monomer dimetakrilat ilavesiyle PS lateksleri hazırlanmıştır. PAAM hazırlanması için monomer; akrilamid (AAm), başlatıcı; amonyum persülfat (APS) ve çapraz bağlayıcı; metilenbisakrilamid (BIS), kullanılırken Sodyum Aljinat için Alginik asit sodyum tuzu Merck tarafından sağland. PAAm polimeri, $7.0 \mathrm{mM}$ APS bileşimi ile desitile su içinde 0.5 M AAm çözündürüldü. 15 dakika boyunca nitrojen ile oksijeni giderildikten sonra $60^{\circ} \mathrm{C}^{\prime}$ de 1 sitılmıştır. Alginatın $\mathrm{T}_{\mathrm{g}}$ si ise, DSC ile $29^{\circ} \mathrm{C}^{\prime}$ de bulundu. $100 \mathrm{~mL}$ solüsyonlarda\% 2.4 PS ve\% $1 \mathrm{Alg}$ hazırland1. Tüm numunelerden su buharlaştırıldıktan sonra bir cam plaka üzerinde PS/Alg ve PS/PAAM kompozitlerinin bileşiminden iki film seti hazırlandı ve kurutulduktan sonra, tüm filmler ayrı ayrı 10 dakika süreyle kızılötesi 1sıtma ile tavlandı. 

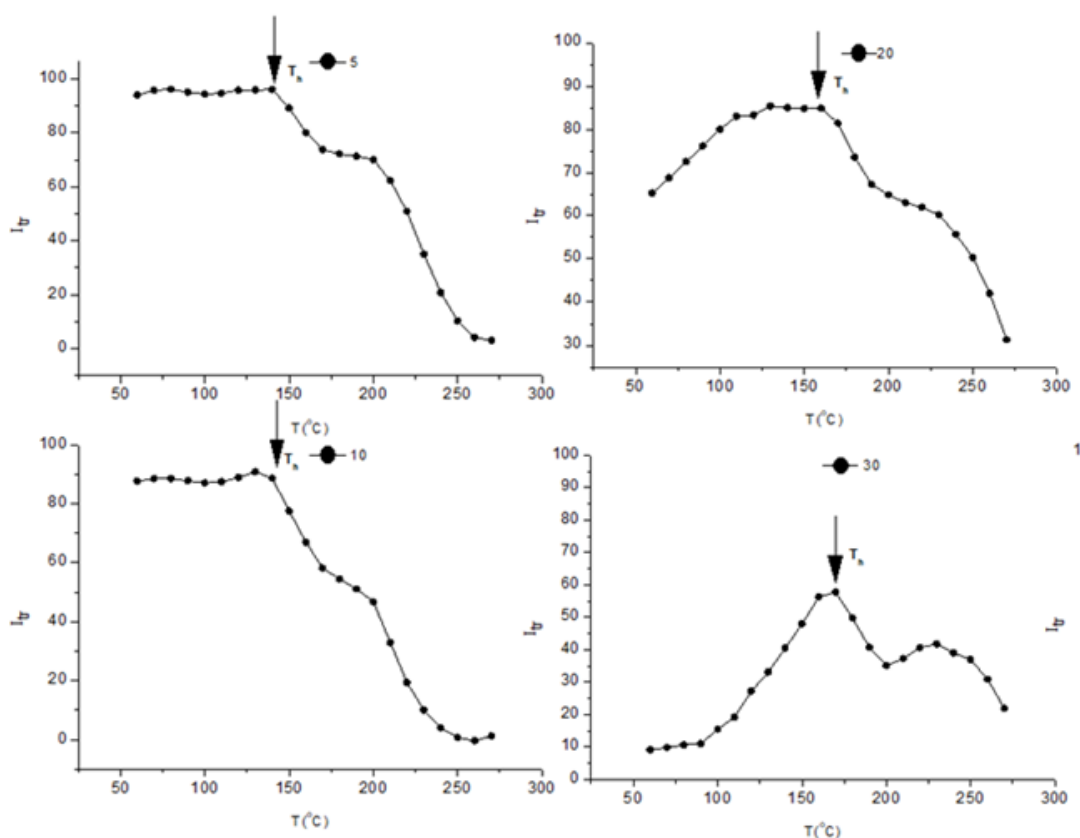

\section{PS/Alg}

Şekil 2: PS/Alg kompozit filmlerinin $\mathrm{I}_{\text {tr }}$ nin $\mathrm{T}\left({ }^{0} \mathrm{C}\right)$ ' ye bağlı değişim grafiği. 5 ila 40 arasındaki sayılar, PS (ağırlık,\%) anlamına gelmektedir.


Şekil 3: PS/PAAM kompozit filmlerinin $\mathrm{I}_{\text {tr }}$ nin $\mathrm{T}\left({ }^{0} \mathrm{C}\right)$ ' ye bağlı değişim grafiği. 5 ila 40 arasındaki sayılar, PS (ağırlık,\%) anlamina gelmektedir.

Şekil 2'de $\mathrm{I}_{\text {tr }}$, belirli bir başlangıç sıcaklı̆̆ı̆ndan (film oluşumu için minimum sicaklık) sonra artar ve tavlama sıcaklığının artmasıyla daha sonra maksimuma ( $\mathrm{T}_{\mathrm{h}}$ kaynaşma sıcaklığı) ulaşır. Bu kritik sıcaklıktan sonra $\mathrm{I}_{\text {tr }}$ tekrar düşmeye başlar. Şekilden anlaşılacağı gibi: (i) kompozit filmdeki PS miktarı artırıldığında, tüm filmlerde $I_{t r}$ 'de bir azalma görülmüştür. $\mathrm{Bu}$ durum, filmdeki boşlukların saçıcı merkez noktaları gibi davranmasına dayandırılarak açıklanmaktadır. Düşük PS katkılı filmlerde faz ayrışımın olduğu lokalize kümelenmeler oluşmuştur (bkz. Şekil 2 ve Şekil 3). PS parçıkları Alginate tarafından tutularak boşluklar içinde rahat hareket etmeleri engellenmektedir. Belirli bir miktar konsantrasyona kadar, bu boşluklar işığın filmden geçtiği yerdeki saçılma merkezlerinin sayısını arttırır[20-23]. Sicaklıkların başlangıcında $\mathrm{I}_{\mathrm{tr}}, 5,10$ ve $20 \mathrm{PS}$ (ağırlıkça\%) konsantrasyonları için yüksektir ve hemen hemen sabittir. Daha sonra artan sicaklikla birlikte azalmaya başlar. 5,10 ve 20 PS (ağırlıkça\%) için yüksek $\mathrm{I}_{\mathrm{tr}}$ 'nin nedeni PAAM parçacıklarının boyutuna bağlanabilir. PAAm parçacıklarının boyutu, Şekil 1 (a) 'da görüldüğü gibi geçen ışı̆̆ın dalga boyundan daha büyüktür. Bu durum, geçen ışı için kompozit filmin neredeyse homojen bir alana dönüşmesine neden olur. Şekil 6'dan görülebileceği gibi, film IR 1sıtmaya maruz bırakıldığında, PAAm parçacıklarının şekli değişir ve parçacıklar küçülmeye başlar. Bu azalma daha fazla boşluk yaratır ve bu boşluklardan 1şık saçılır, bu da 5, 10 ve 20 PS (ağırlık,\%) filmler için Itr'de azalma ile sonuçlanır. 

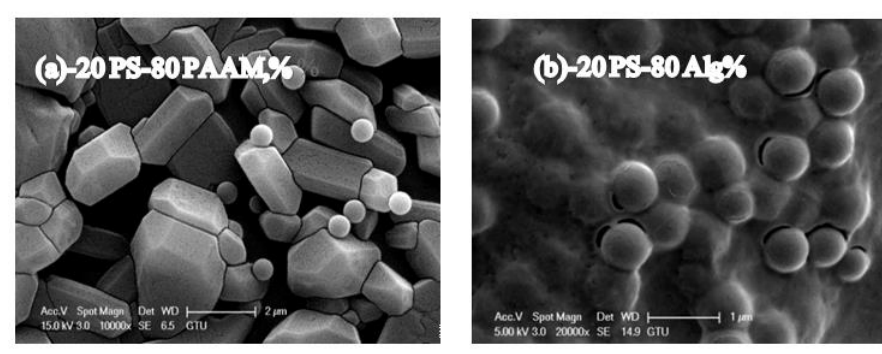

Şekil 4: (a)PAAM içnde (b) Alg içinde \%20 PS katkılı filmlerin SEM görüntüsü.

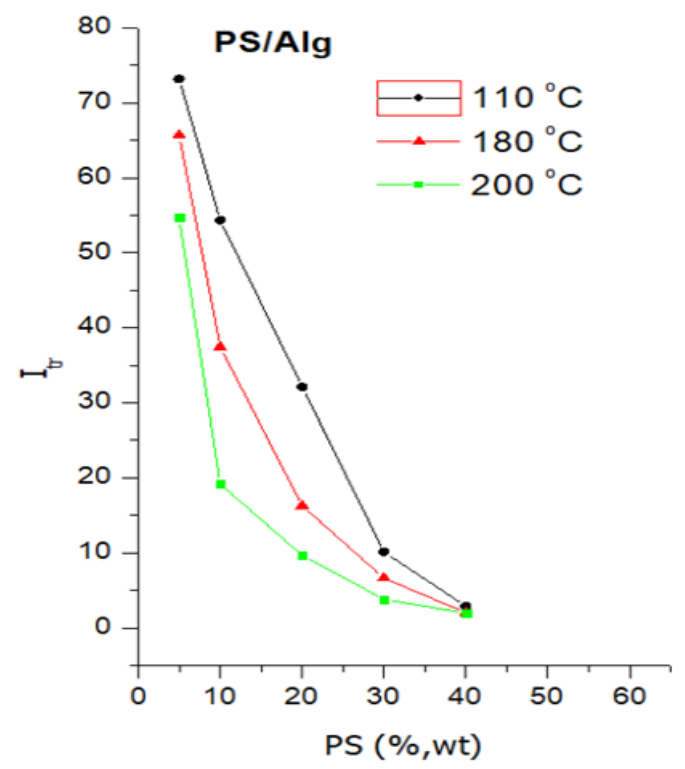
grafiği

Şekil 5: $\mathrm{I}_{\text {tr }}$ nin \%PS ye göre 110,180 ve $180{ }^{\circ} \mathrm{C}$ deki değişim

PS miktarına göre $\mathrm{I}_{\text {tr }}$ değişimleri Şekil 5 'te sunulmuştur. $\mathrm{I}_{\text {tr }}$, 110,180 ve $200{ }^{\circ} \mathrm{C}^{\prime} \mathrm{de}$ tüm kompozitler için azalmaktadır. Bu sıcaklıklarda hareket eden PS parçacıkları, matriste yeni ve daha büyük boşluklar oluşturan yeni kümeler oluşturur. Parçacıkların bu şekilde yer değiştirmesinin bir sonucu olarak, Itr azalır. Daha yüksek sıcaklıklarda(belirli bir sıcaklığa kadar), PS yüzdesinin artmasıyla $\mathrm{I}_{\text {tr }}$ artmaktadır(Şekil 2). Burada, PS miktarı arttığında, PS parçacıkları matriste boşlukları doldurur ve oluşan az boşluklu yapı da yüksek $I_{\text {tr }}$ nin olmasına neden olur. Şekil 6'da, minimum film oluşum sıcaklığı; $T_{o}$ [24] kaynaşma sıcaklığ $1 T_{h}$ değerleri kompozit filmler için de belirlendi. $\mathrm{Bu}$ kritik $\mathrm{T}_{\mathrm{o}}$ sıcaklığının altında, lateks opak formdadır ve bu sicaklığın üzerindeki filmler daha fazla 1sitmayla homojen hale gelmeye başlar [25]. $\mathrm{T}_{\mathrm{h}}$ [18], kompozit filmlerin kaynaşma sıcaklığını tanımlamak için kullanılır. Boşluk kapanmasından sonra, interdiffusion işlemi $T_{h}$ değerlerinin üzerinde başlar ve burada kütle merkezleri ara yüzeyi boyunca dönüşüm için yeterli kinetik enerji kazanarak yer değiştirirler. Şekil 6 (a ve b) 'dan görüldüğü gibi, PS miktarının eklenmesiyle $\mathrm{T}_{\mathrm{o}}$ ve $\mathrm{T}_{\mathrm{h}}$ değerleri de artar. $\mathrm{Bu}$, $\mathrm{T}_{\mathrm{o}}$ 'ınn yüksek $\mathrm{T}_{\mathrm{g}}{ }^{\mathrm{y}} \mathrm{ye}$ sahip $\mathrm{PS}$ miktarından etkilendiğini göstermektedir. Düşük katkılı 5 ve 10 PS (\%,wt) filmler için, $T_{o}$ çok fazla değişmez ve bu PS miktarının üzerinde, $\mathrm{T}_{\mathrm{o}}$ artar.
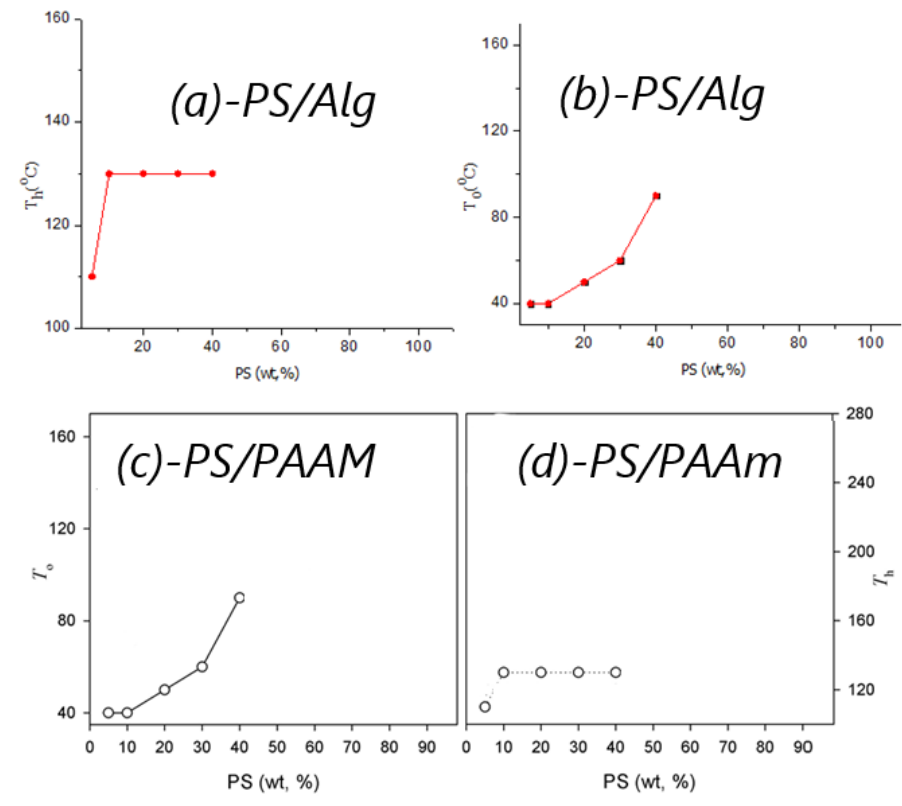

Şekil 6: (a) PS/Alg kompozit filmleri için Kaynaşma sıcaklığ $T_{h} \operatorname{nin}(b)$ Minimum film oluşum sicaklığı $T_{o}$ un PS ye göre (c) Minimum film oluşum sıcaklığ $T_{o}$ un (d) PS/PAAM kompozit filmleri için Kaynaşma sıcaklığ $1 T_{h}$ nin PS ye göre değişim grafiği.

\section{Aktivasyon Enerjilerinin Hesaplanması}

Boşluk kapanması ve interdifüzyon süreçleri için aşağıdaki denklemler kullanılmıştır [18]

$$
\begin{gathered}
I_{t r}(T)=S(t) \exp \left(-\frac{3 \Delta H}{k T}\right) \\
I_{t r}(T) / I_{t r}(\infty)=A \exp \left(-\frac{\Delta E}{2 k_{B} T}\right)
\end{gathered}
$$

$\mathrm{S}(\mathrm{t})=(\mathrm{yt} / 2 \mathrm{AC})^{3}, \mathrm{k}$ Boltzmann's sabitidir. $\Delta \mathrm{H}$, boşluk kapama $\Delta \mathrm{E}$,interdifüzyon enerjileridir. $\mathrm{I}_{\mathrm{tr}}, \quad 800 \quad \mathrm{~nm} \quad$ deki 1 ş1k geçirgenliğidir. Bu denklemler kullanılarak hesaplana aktivasyon enerjilerinin ğrafiği şekil 7 de sunulmuştur. Itr'deki artış boşluk kapatma işlemi ile açıklanmaktadır, $\Delta \mathrm{H}$ aktivasyon enerjileri $\mathrm{T}_{0}$ ve $T_{h}$ arasındaki bölgeye denklem 1 uygulanarak bulunmuştur. Bu değerler Şekil 7 (a) ve Şekil 7 (c) 'de görülmektedir. Film oluşumu için gerekli kuvvetparçacık boyutunun [26] tersine bağlıdır. Düşük sıcaklıkların etkili olan boşluk doldurma işlemi sırasında yüksek aljinat miktarının olduğu kompozitler için viskoz akışın aktivasyon enerjisi daha azdır.

Düşük PS lateks miktarı için, $\Delta \mathrm{H}$ değerleri daha küçüktür çünkü Alg ve PAAM, PS parçacıklarından daha düşük $T_{\mathrm{g}}$ 'ye ve daha büyük yüzey alanına sahiptir. $\Delta H$, PS'nin yüksek $\mathrm{T}_{\mathrm{g}}$ 'si nedeniyle artar. Şekil 7 (b) 'de, kompozit filme PS lateksinin eklenmesi ile $\Delta E^{\prime}$ 'nin arttığ , yani film oluşumu için fazla enerji ile gerçekleştirilebileceğini göstermektedir. $\mathrm{Bu}$ nokta, PS parçacıklarının $\mathrm{T}_{\mathrm{g}}$ 'si ile ilgilidir. PS lateks ilavesi ile polimer zincirlerinin hareketi için gerekli enerji artacaktır. Tek bir zincir, polimer-polimer ara yüzündeki difüzyonun üstesinden gelmek için viskoz akış süreci ile elde edilenden daha fazla enerjiye 
ihtiyaç duyduğundan, interdiffüzyon enerjileri boşluk kapatma aktivasyon enerjilerinden çok daha büyüktür.
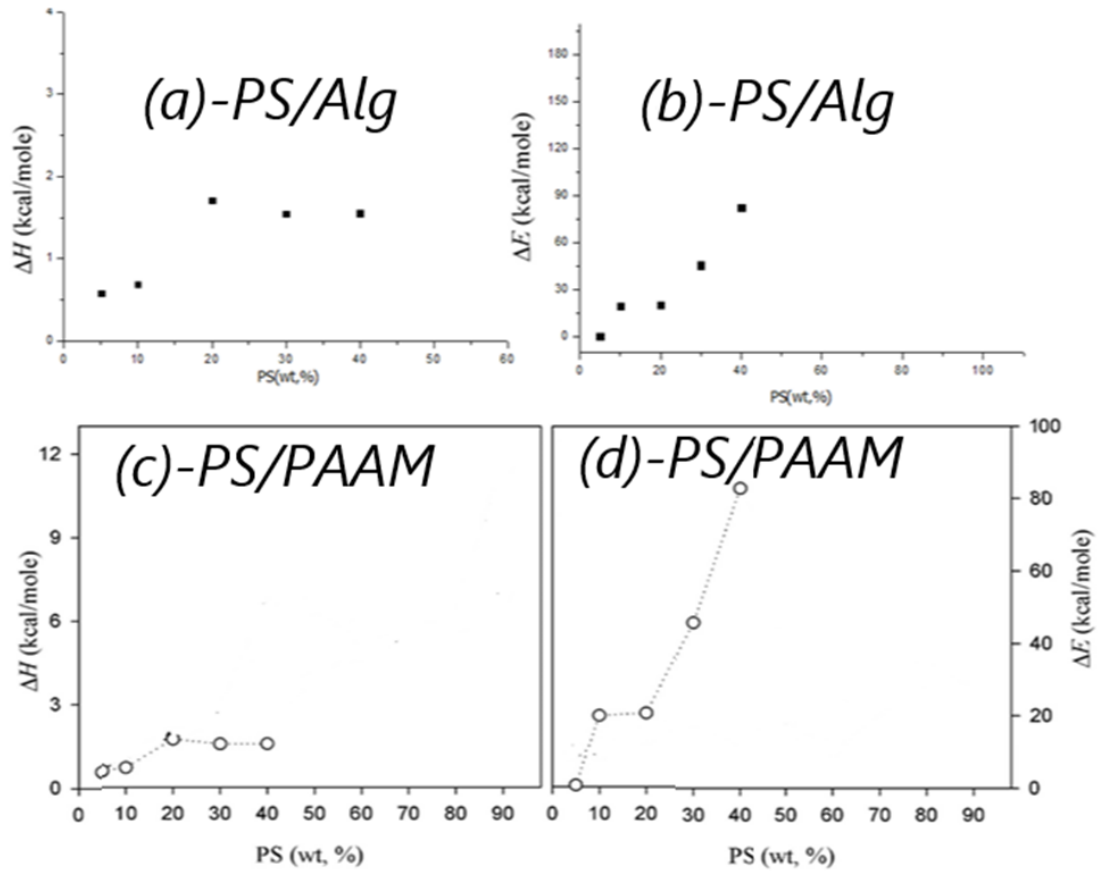

Şekil 7: PS/Alg için Boşluk kapanması $(\Delta \mathrm{H})$ ve interdiffusion $(\Delta \mathrm{E})$ enerjileri (a ve b) ve PS/PAAM kompozitleri için (c ve d). ile PS (ă̆ırlıkça\%) değişim grafikleri

\section{Sonuç}

Bu çalışmada, infrared ısıtma altında PS/Alg ve PS/PAAM kompozit matrisinden film oluşumunu izlemek için basit ve kullanışlı bir yöntem olan UV-Visible tekniği ile görünür bölgede geçen 1 ş1k şiddetinin izlenmesi suretiyle spektroskopik bir teknik uygulanmıştır. Sonuçlar, film oluşum sürecinin düşük sıcaklıklarda gerçekleştiğini gösterdi. Kompozit filmlerin aktivasyon enerjileri, her iki kompozit sistem için benzer davranış ve sonuçlar gösterdi. Bu sonuç, Alg ve PAAM parçacıklarının düşük $\mathrm{T}_{\mathrm{g}}$ sine dayandırılabilir. Her iki kompozit sistem için interdifüzyon enerjilerinin boşluk kapatma enerjilerinden daha yüksek olduğu bulundu. $\mathrm{Bu}$, matristeki karşılaşılan potansiyel engelinin üstesinden gelmek için tek bir zincirin tüm viskoz akıştan daha yüksek enerjiye ihtiyaç duyması oldukça anlaşılır bir durum olarak değerlendirildi.

\section{Kaynakça}

1. Mabbett I. Elvins J. Gowenlock C. Jones P. Worsley D. (2013) Effects of highly absorbing pigments on near infrared cured polyester/melamine coil coatings Progress in Organic Coatings 76(9) 1184-1190.

2. Jeong H.E., Kwak M.K., and Suh K.Y. (2010). Stretchable, adhesion-tunable dry adhesive by surface wrinkling, Langmuir, (26), 2223-2226.

3. Pekcan O. and Arda E. (1999). Void closure and interdiffusion in latex film formation by photon transmission and fluorescence methods, Colloids and Surfaces A: Physicochemical and Engineering Aspects., (153), 537-549.

4. Fyrillas M., Georgiou G., Vlassopoulos D., and Hatzikiriakos S. (1999). Polymer Engineering \& Science., 39.
5. Sperry P.R., Snyder B.S., O’Dowd M.L., and Lesko P.M. (1994). Role of Water in Particle Deformation and Compaction in Latex Film Formation, Langmuir, (10), 2619-2628.

6. Winnik Mitchell A. (2002). Interdiffusion and crosslinking in thermoset latex films A Phenomenological Theory of Sintering, Journal of Coatings Technology., (74), 49-63.

7. Yoo J.N., Sperling L.H., Glinka C.J., and Klein A. (1991). Characterization of film formation from polystyrene latex particles via SANS. 2. High molecular weight, Macromolecules Macromolecules, (24), 2868-2876.

8. Pekcan O. (1994). Interdiffusion of polymer chains during latex coating Polymer for Advanced Technologies, 5(9), 479-484.

9. Eckersley S.T., and Rudin A. (1994). Drying behavior of acrylic latexes Progress in Organic Coatings, (23), 387-402.

10. Cannon L.A., and Pethrick R.A. (1999). Effect of the GlassTransition Temperature on Film Formation in 2-Ethylhexyl Acrylate/Methyl Methacrylate Emulsion Copolymers Macromolecules, (32), 7617-7629.

11. Ugur S. (2006). Fluorescence Study of F1lm Formation From Hard/ Soft Latex Blends, e-polymers, no-037.

12. Pekcan O., Winnik M.A., and Croucher M.D. (1990). Fluorescence studies of coalescence and film formation in poly (methyl methacrylate) nonaqueous dispersion particles Macromolecules, (23), 2673-2678.

13. Ugur S., and Pekcan O. (2005). Time evolution of film formation from polystyrene particles: A percolation approach, Colloid and Polymer Science., (284), 309-316.

14. Keddie J.L., (1997). Film formation of latex Macromolecules, (21), 101-170.

15. Arda E., Bulmus V., Piskin E., and Pekcan O. (1999). Molecular weight effect on latex film formation from 
polystyrene particles: a photon transmission study, Journal of Colloid Interface Science, (213), 160-168.

16. Arda E., and Pekcan O. (2001). Time and temperature dependence of void closure, healing and interdiffusion during latex film formation Polymer, (42), 7419-7428.

17. Arda E., Ozer F., Piskin E., and Pekcan O. (2001). Film formation from nanosized copolymeric latex particles: a photon transmission study, Journal of Colloid Interface Science., (233), 271-279.

18. Pekcan O., and Arda E. (2000). Packing effect on latex film formation: A photon transmission study Polymer International, 49(7), 678 - 684.

19. Voyutskii S.S., (1963) Autohesion and Adhesion of High Polymers, (Polymer Reviews Vol. 4) Interscience, New York.

20. Voyutskii S.S., Starkh B.W., (1954) Physical Chemistry of Film Formation from High Polymer Dispersions, Moscow.

21. Yang Y., and Gupta M.C. Dudley KL, Lawrence RW. (2005). Novel Carbon Nanotube-Polystyrene Foam Composites for Electromagnetic Interference Shielding, Nano Letters, (5), 2131-2134.

22. Afzal A., Kausar A., and Siddiq M. (2016). Perspectives of Polystyrene Composite with Fullerene, Carbon Black, Graphene, and Carbon Nanotube: A ReviewPolymerPlastics Technology and Engineering, (55), 1988-2011.

23. Thakur V.K., Thakur M.K., Raghavan P., and Kessler M.R. (2014). Progress in Green Polymer Composites from Lignin for Multifunctional Applications: A Review, ACS Sustainable Chemistry \& Engineering, (2), 1072-1092.

24. Yargi O, Ugur S, Pekcan O. (2014). Polymer/Carbon Nanotube Composite Film Formation: A Fluorescence Study, Polymer Composites, 35(5), 817-826.

25. Tepehan F., Sunay S., Uğur Ş. and Pekcan O (2007). Film formation from $\mathrm{TiO} 2$-polystyrene latex composite: a fluorescence study, Composite Interfaces, 14(3), 243-260.

26. Ugur S, Salman O.U., Tepehan G., Tepehan F., Pekcan O. (2005). Fluorescence Study on Al2O3-Polystyrene LatexComposite Film Formation, Polymer Composites, 26(3), 352-360. 